\title{
Transforming growth factor- $\beta /$ miR-143-3p/cystatin B axis is a therapeutic target in human ovarian cancer
}

\author{
WENCAI GUAN ${ }^{1}$, XINGXING WANG $^{1}$, QUNBO LIN ${ }^{1}$, JINGUO ZHANG $^{1,2}$, \\ WEIMIN REN ${ }^{1,2}$ and GUOXIONG XU ${ }^{1,2}$ \\ ${ }^{1}$ Research Center for Clinical Medicine, Jinshan Hospital, Fudan University, Shanghai 201508; \\ ${ }^{2}$ Department of Oncology, Shanghai Medical College, Fudan University, Shanghai 200032, P.R. China
}

Received March 4, 2019; Accepted May 24, 2019

DOI: $10.3892 /$ ijo.2019.4815

\begin{abstract}
We previously reported that cystatin B (CSTB) is a progression marker of human ovarian cancer (OC); however, the regulatory mechanism of CSTB and its function in OC remain unclear. The present study aimed to explore the mechanism underlying transforming growth factor- $\beta$ (TGF- $\beta$ ) 1-mediated CSTB regulation, and to examine the function of CSTB on OC cell proliferation and apoptosis. Using the online program, miRWalk, a microRNA (miR)-143-3p was detected, which contains a homologous sequence of the potential binding site to the 3'-untranslated region (3'-UTR) of CSTB. A dual-luciferase reporter assay confirmed the interaction between miR-143-3p and CSTB 3'-UTR. Treating OC cells with miR-143-3p mimics or inhibitors resulted in a decrease or an increase of CSTB expression at mRNA and protein levels, respectively. Additionally, CSTB was significantly overexpressed, whereas miR-143-3p was downregulated in human OC tissues compared with normal ovarian tissues. A negative correlation between miR-143-3p and CSTB mRNA expression was observed in ovarian malignant tumors. The levels of primary and mature miR-143-3p expression were upregulated in OC cells after TGF- $\beta 1$ treatment; the action of TGF- $\beta 1$ was abolished in the presence of an inhibitor of TGF- $\beta$ type I receptor. These results indicated an axis between TGF- $\beta$, miR-143-3p and CSTB in OC cells. Furthermore, high levels of CSTB expression were associated with the poor overall survival of patients with OC. Knockdown of CSTB
\end{abstract}

Correspondence to: Professor Guoxiong Xu, Research Center for Clinical Medicine, Jinshan Hospital, Fudan University, 1508 Longhang Road, Shanghai 201508, P.R. China

E-mail: guoxiong.xu@fudan.edu.cn

Abbreviations: 3'-UTR, 3'-untranslated region; CSTB, cystatin B; EMT, epithelial to mesenchymal transition; miR, microRNA; TGF- $\beta$, transforming growth factor- $\beta$; OC, ovarian cancer; RT-qPCR, reverse transcription-quantitative polymerase chain reaction

Key words: cystatin B, epithelial ovarian cancer, microRNA, proliferation, stefin $\mathrm{B}$, TGF- $\beta$ signaling pathway resulted in a decrease in OC cell proliferation and arrested cells in G2/M phase. In addition, suppression of CSTB induced cell apoptosis. In conclusion, CSTB was overexpressed and miR-143-3p was downregulated in ovarian malignant tumors. Mature miR-143-3p directly bound CSTB 3'-UTR, leading to a decrease in CSTB expression in OC cells, which was regulated by TGF- $\beta 1$. Our findings suggest the potential therapeutic application of targeting the TGF- $\beta / \mathrm{miR}-143-3 \mathrm{p} / \mathrm{CSTB}$ axis for treating patients with $\mathrm{OC}$.

\section{Introduction}

Cystatin B (CSTB, stefin B) is an endogenous inhibitor of intracellular cysteine proteases $(1,2)$. Previously, we reported that CSTB is a progression marker of human epithelial ovarian cancer (OC) (3), a disease ranked as the eight most common cancer worldwide in females (4) and gynecological cancer with the highest rate of mortality in the United States of America (5). CSTB has been implicated in inflammation (6), autosomal recessive disorders (7), and cancer (3,8-11); however, the regulatory mechanism and the function of CSTB on cell proliferation in epithelial OC (EOC) remains unknown.

MicroRNAs (miRNAs/miRs) are a class of small noncoding RNA of $\sim 22$ nucleotides in length that can regulate a gene by binding to its 3'-untranslated region (3'-UTR), triggering the degradation of mRNA or the suppression of protein translation $(12,13)$. Accumulating evidence suggests that numerous miRNAs of multi-gene regulatory capacity are dysregulated in cancer $(14,15)$, and have been associated the regulation of biological processes, such as carcinogenesis, metastasis, epithelial-mesenchymal transition (EMT) and chemoresistance in EOC $(16,17)$, and are regulated by cytokines $(18,19)$.

Transforming growth factor (TGF)- $\beta$ belongs to a superfamily of secreted cytokines and serves a key role in many cellular processes. Members of the TGF- $\beta$ superfamily transmit signals via the Smad-dependent and -independent pathways $(20,21)$. The canonical pathway of TGF- $\beta$ signaling occurs through the activation of its corresponding membrane serine/threonine kinase receptors (type I and type II receptors) to activate the intracellular signaling transducer $\operatorname{Smad} 2 / 3$ proteins, which form a complex with Smad4 (22). After the Smad-complex translocates into the nucleus, it acts as a transcription factor to regulate the expression of a target gene (21). Smad-independent 
pathways, such as the MAPK and PI3K signaling pathways, can also be induced by TGF- $\beta$ to initiate signal transduction and gene regulation (23). The Smad-dependent and -independent pathways have been demonstrated to contribute to the pathogenesis of OC $(24,25)$. Our previous study has revealed that CSTB is mediated by the TGF- $\beta$ signaling pathway (3); however, the regulatory mechanism by which TGF- $\beta 1$ regulates CSTB and its function in OC remain unclear.

The present study aimed to explore the mechanism underlying TGF- $\beta 1$-mediated CSTB regulation via miR-143-3p, and examine the function of CSTB on OC cell proliferation and apoptosis.

\section{Materials and methods}

Bioinformatics analyses. To predict the miRNAs targeting CSTB, miRWalk (version 1.0; http://zmf.mm.uni-heidelberg. de/apps/zmf/mirwalk/predictedmirnagene.html) was applied. To analyze the expression of CSTB mRNA and miR-143-3p between normal ovarian tissues and ovarian malignant tumors, three gene expression datasets (GSE36668, GSE40595, GSE63885) for CSTB and one microRNA expression dataset (GSE47841) for miR-143-3p were downloaded from the public Gene Expression Omnibus (GEO) database (http://www. ncbi.nlm.nih.gov/geo/). All three CSTB mRNA expression profiles from 12 normal ovary samples and 108 ovarian serous carcinoma samples were generated by the Affymetrix Human Genome U133 Plus 2.0 Array and a miR-143-3p expression profile from 9 normal ovary samples and 12 ovarian serous carcinoma samples was generated by the Affymetrix Multispecies miRNA-2 Array. These transcriptome microarray datasets were integrated and analyzed by $\mathrm{R}$ software version 3.4.2 (https://www.r-project.org/). Additionally, to analyze the differential expression of CSTB between ovarian surface epithelium and ovarian serous carcinoma, the online Oncomine tool (www.oncomine.org) was used. In this analysis, the median levels of CSTB mRNA expression were calculated from two datasets: $\mathrm{Lu}$ (26) and Bonome (27). To analyze the overall survival (OS) of patients with OC, the Kaplan-Meier Plotter database (www.kmplot.com) was employed. Patients with OC selected for OS analysis were divided into two groups according to the cutoff value set as the median expression levels of CSTB. The OS data were presented as a survival plot, and analyzed with a log rank test.

Patients and ovarian tissue samples. A total of 35 fresh ovarian tissues samples were obtained from patients (age range: 24-87 years) who underwent surgery at Jinshan Hospital from January 2012 to December 2015 (11 normal ovarian samples from patients with non-ovarian tumor and 24 ovarian serous tumor samples, including 10 benign, 3 borderline, and 11 malignant tumors). All tissue samples were immediately snapped frozen in liquid nitrogen and stored at $-80^{\circ} \mathrm{C}$ until use. None of the patients had received chemotherapy or radiotherapy prior to surgery. The present study was approved by the Ethics Committee of Jinshan Hospital, Fudan University.

Cell culture and TGF- $\beta$ treatment. The human OC cell lines OVCAR-3 and SK-OV-3 cells were obtained from American Type Culture Collection (ATCC) and maintained in RPMI-1640 and Dulbecco's modified Eagle's media (Corning Inc.), respectively, with $10 \%$ (v/v) fetal bovine serum (Invitrogen; Thermo Fisher Scientific, Inc.). Cells were cultured as described previously (24). For TGF- $\beta 1$ treatment, after seeding cells for $24 \mathrm{~h}, 10 \mathrm{ng} / \mathrm{ml}$ recombinant human TGF- $\beta 1$ (cat. no. 240-B, R\&D Systems, Inc.) was added and incubated at $37^{\circ} \mathrm{C}$ for $3 \mathrm{~h}$. Cells without any treatment were used as a blank control. For blocking of the TGF- $\beta 1$ signaling pathway, cells were pre-treated with $10 \mu \mathrm{M}$ SB431542, a TGF- $\beta$ type I receptor inhibitor (Sigma-Aldrich; Merck KGaA) for $30 \mathrm{~min}$, followed by $10 \mathrm{ng} / \mathrm{ml} \mathrm{TGF}-\beta 1$ treatment at $37^{\circ} \mathrm{C}$ for $3 \mathrm{~h}$.

Total RNA extraction and reverse transcription-quantitative PCR (RT-qPCR). Total RNA was extracted from cells or tissue samples using TRIzol reagent (Thermo Fisher Scientific, Inc.) according to the manufacturer's protocols. Primary miRNA, mature miRNA and mRNA were reversely transcribed using a Transcriptor First Strand cDNA Synthesis Kit (Roche Applied Science) according to the manufacturer's instructions. The primer sequences were listed in Table S1. RT-qPCR was performed at $95^{\circ} \mathrm{C}$ for $10 \mathrm{~min}$, followed by 40 cycles of $95^{\circ} \mathrm{C}$ for $10 \mathrm{sec}$ and $60^{\circ} \mathrm{C}$ for $30 \mathrm{sec}$ using the FastStart Universal SYBR-Green Master (Roche Applied Science) on an ABI PRISM 7300 Real Time PCR System (Applied Biosystems; Thermo Fisher Scientific, Inc.). The relative expression of mRNA, primary miRNA or mature miRNA was evaluated using the $2^{-\Delta \Delta \mathrm{Cq}}$ method (28) and all experiments were conducted in triplicate. 18S and U6 was used as an internal control for mRNA and miRNA, respectively.

Lentivirus-delivered short hairpin RNA (shRNA) infection, siRNA and miRNA mimics/inhibitors transfection. Lentiviral vectors containing shRNA for CSTB (CSTB-shRNA, sh-CSTB) and negative control (NC-shRNA, sh-NC) were obtained from Shanghai GenePharma Co., Ltd. Three specific CSTB-siRNAs (5'-GUCCCAGCUUGAAGAGAAATT-3' for si-CSTB-1, 5'-GGACAAACUACUUCAUCAATT-3' for si-CSTB-2, and 5'-CCCUUGACCUUAUCUAACUTT-3' for si-CSTB-3) and a negative control (NC-siRNA, si-NC) were synthesized by Shanghai GenePharma Co., Ltd. The miR-143-3p mimics, miR-negative control (miR-NC), miR-143-3p inhibitor (anti-miR-143), and the negative control of inhibitor (anti-miR-NC) was obtained from Guangzhou RiboBio Co., Ltd. (Table S1). After seeding at $2 \times 10^{5}$ cells/well in 6-well plates and incubating for $24 \mathrm{~h}$, cells were transfected with $50 \mathrm{nM}$ si-CSTB or si-NC, miR-143-3p mimics or miR-NC, 200 nM miR-143-3p inhibitors or miR-NC using X-tremeGENE siRNA Transfection Reagent (Roche Applied Science). Cyanine 3 (Cy3) dye-labeled miR-NC was used to evaluate the transfection efficiency detected by fluorescence microscopy. The sequence of si-CSTB-2 was used for subsequent experimentation. Knockdown of CSTB was performed in OC cells by infecting cells with CSTB-shRNA lentivirus according to the manufacturer's protocols. Briefly, after cells were plated in 6-well plates and incubated at $37^{\circ} \mathrm{C}$ for $24 \mathrm{~h}$, the amount of lentiviral particle solution (multiplicity of infection=20) was added into the culture media containing $8 \mu \mathrm{g} / \mathrm{ml} /$ well Polybrene for $24 \mathrm{~h}$. The media was replaced with fresh media containing $2 \mu \mathrm{g} / \mathrm{ml}$ puromycin for selection every few days. Following puromycin selection for several passages, the 
detection of protein knockdown or the phenotypic assay were performed.

Western blotting. Cells were lysed in radioimmunoprecipitation assay lysis buffer (Thermo Fisher Scientific, Inc.) with $1 \%$ phenylmethanesulfonyl fluoride (Beyotime Institute of Biotechnology) and 1\% phosphatase inhibitor (Nanjing KeyGen Biotech Co., Ltd.), followed by sonication. The protein was extracted from the supernatant after $20 \mathrm{~min}$ of $18,000 \mathrm{x}$ g centrifugation at $4^{\circ} \mathrm{C}$. Proteins were quantified via a BCA assay. Equal amount proteins were separated on $15 \%$ SDS-PAGE and transferred to a polyvinylidene difluoride membrane (EMD Millipore). After blocking with 5\% non-fat milk in Tris-buffered saline with Tween 20 at room temperature for $1 \mathrm{~h}$, the membrane was incubated with a primary antibody at $4^{\circ} \mathrm{C}$ overnight and subsequently incubated with horseradish peroxidase-conjugated goat anti-rabbit or anti-mouse IgG (cat. nos. 7074 and 7076, respectively; 1:5,000 dilution; Cell Signaling Technology, Inc.) for $1 \mathrm{~h}$ at room temperature. The following primary antibodies were used: Rabbit anti-CSTB (1:5,000 dilution; Abcam) and mouse anti- $\beta$-actin (1:5,000 dilution; ProteinTech Group., Inc.). Signals were detected using Immobilon ${ }^{\mathrm{TM}}$ Western Chemiluminescent HRP Substrate (EMD Millipore) and quantified using Tanon-4500 Gel Imaging System with GIS ID Analysis Software v4.1.5 (Tanon Science and Technology Co., Ltd.).

Plasmid construction and dual-luciferase reporter assay. The whole 3'-UTR of CSTB that contains the binding site of miR-143-3p predicted by the miRWalk program (http://mirwalk.umm.uni-heidelberg.de/) was amplified from genomic DNA using the Pfu Ultra II Fusion HS DNA Polymerase (Stratagene; Agilent Technologies, Inc.) with well-designed primers (Table S1). After the dual-luciferase reporter vector $\mathrm{pEZX-FR2} \mathrm{(GeneCopoeia,} \mathrm{Inc.)} \mathrm{was} \mathrm{linearized}$ by EcoRI and XhoI restriction enzymes, the PCR product was inserted into pEZX-FR2 using the EasyGeno Assembly Cloning Kit (Tiangen Biotech Co., Ltd.) to construct a wild-type clone named as CSTB-3UTR-wt. A mutation was induced in the consensus sequence of the miR-143-3p binding site in the 3'-UTR of CSTB using the QuikChange II Site-Directed Mutagenesis Kit (Stratagene; Agilent Technologies, Inc.) to construct a mutated clone named as CSTB-3UTR-mut. All clones were verified by restriction enzymes EcoRI and XhoI digestion and DNA sequencing with primer (5'-GATCCG CGAGATCCTGAT-3') by GENEWIZ, Inc. For the dual-luciferase reporter assay, 293T cells (ATCC) were cultured in 24-well plates. Once subconfluent, the cells were transfected with CSTB-3UTR-wt or CSTB-3UTR-mut plasmid $(0.5 \mu \mathrm{g})$, plus miR-143-3p mimics, inhibitors or their negative controls using Roche X-tremeGENE siRNA Transfection Reagent $(2 \mu \mathrm{l})$. After transfection for $24 \mathrm{~h}$, the cells were lysed and luciferase activities were determined using the Luc-Pair ${ }^{\mathrm{TM}}$ Duo-Luciferase Assay Kit (GeneCopoeia, Inc.) according to the manufacturer's instructions. Renilla luciferase activity was used for the normalization of Firefly luciferase activity.

Cell proliferation assay and cell cycle detection. For the cell proliferation assay, NC-shRNA- or CSTB-shRNA-infected OVCAR-3 cells were plated in 96-well plates at a density of
$3 \times 10^{3}$ cells/well. Cell proliferation was measured at 24,48 , and $72 \mathrm{~h}$ post-infection by the Cell Proliferation Reagent (Cell Counting Kit-8; Dojindo Molecular Technologies, Inc.) according to the manufacturer's protocols. The signal in optical density was read by a microplate reader (BioTek Instruments, Inc.) at $450 \mathrm{~nm}$.

The cell cycle was detected by flow cytometry. Briefly, CSTB-shRNA-infected OVCAR-3 cells were cultured in 6 -well plates for $48 \mathrm{~h}$. The cells were harvested, washed twice with PBS, and fixed in cold $70 \%$ ethanol for $2 \mathrm{~h}$ at $-20^{\circ} \mathrm{C}$. After washing with PBS twice, the cells were resuspended in $500 \mu \mathrm{l}$ of propidium iodide (PI)/RNase Staining Buffer (BD Pharmingen) and incubated in the dark for $15 \mathrm{~min}$ at room temperature. Cells $(15,000)$ were then detected by flow cytometry (Beckman Coulter, Inc.). The data were analyzed using ModFit LT software v4.1.7 (Verity Software House, Inc.), and presented as the percentage of the cell population at the G0/G1, S and G2/M phases. Scramble-shRNA-infected OVCAR-3 cells were used as a control.

Detection of apoptotic cells. Flow cytometry and western blotting were conducted to detect apoptotic cells. Briefly, cells were cultured in 6-well plates for $48 \mathrm{~h}$. After washing with PBS, the cells were collected and stained with $1 \mu$ l Annexin V conjugated with allophycocyanin (BD Pharmingen) and $5 \mu 1$ PI for $15 \mathrm{~min}$ at room temperature. Apoptotic cells were detected by flow cytometry with FlowJo X software v10.0.7r2 (BD Biosciences). The expression of pro-apoptotic protein Bcl-2-associated X protein (Bax) was analyzed by western blotting as aforementioned using an antibody against Bax (1:2,000 dilution; Cell Signaling Technology, Inc.).

Statistical analyses. All experiments were repeated at least three times and all analyses were performed with SPSS 21 for Windows (IBM Corp.). For comparisons between two groups in an experiment, a Student's t-test was applied. For multiple comparisons, one-way analysis of variance was used, followed by a Tukey's post-hoc test. For the correlation analysis between CSTB and miR-143-3p, nonparametric Spearman rank correlation was used. The results were presented as the mean \pm the standard error of the mean. $\mathrm{P}<0.05$ was considered to indicate a statistically significant difference.

\section{Results}

CSTB is negatively correlated with miR-143-3p in human OC. miRNAs which can bind to the CSTB 3'-UTR were screened using web-based programs in the miRWalk database. For targeting CSTB, miR-143-3p was proposed as a potential candidate by 5 out of 10 available prediction programs (Fig. 1A). Additionally, the expression data of transcripts were extracted from GSE36668, GSE40595 and GSE63885 datasets to obtain the mRNA expression profile of CSTB, and GSE47841 dataset for miR-143-3p in an online GEO database using integrated bioinformatics analyses. Upregulated expression of CSTB mRNA was detected in ovarian serous carcinoma $(n=108)$ compared with normal ovary controls $(n=12)$ (Fig. 1B), while miR-143-3p expression was lower in ovarian serous carcinoma $(n=12)$ than normal ovary controls $(n=9)$ (Fig. 1C). As the patients with CSTB mRNA data available in the online datasets 
A

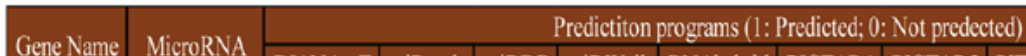

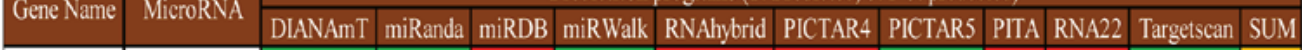

CSTB $\quad$ hsa-miR-143

B



C miR-143-3p expression



D

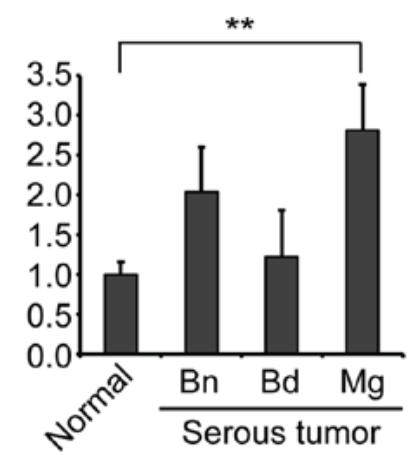

E

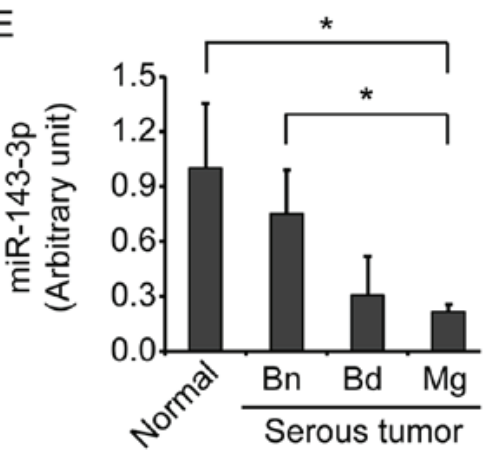

F $\quad r=-0.362 \quad P<0.05$

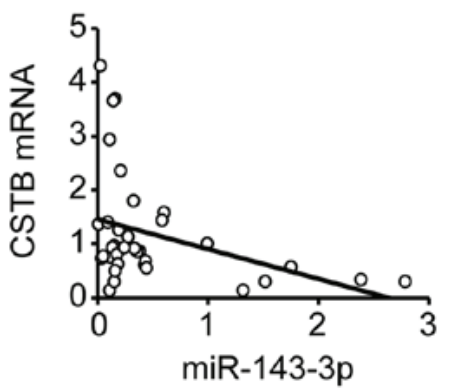

Figure 1. Detection of CSTB mRNA and miR-143-3p expression in human ovarian tissues, and their correlation. (A) Prediction of miR-143-3p binding to CSTB. miR-143-3p was proposed to be a candidate from 5 out of 10 programs in the miRWalk database. (B) Comparison of CSTB mRNA expression between normal ovary controls and ovarian serous carcinoma. Data were obtained from three gene expression datasets (GSE36668, GSE40595, GSE63885) in the GEO database. (C) Comparison of miR-143-3p expression between normal ovary controls and ovarian serous carcinoma. Data were obtained from a miRNA expression dataset (GSE47841) in the GEO database. (D and E) The expression of CSTB mRNA and miR-143-3p was detected by reverse transcription-quantitative PCR in freshly isolated ovarian tissues, including normal ovarian tissues $(n=11), B n(n=10), B d(n=3)$ and Mg $(n=11)$. (F) The correlation between CSTB mRNA and miR-143-3p in human ovarian tissues (total $\mathrm{n}=35$ ). Data are presented as the mean \pm standard error of the mean. ${ }^{*} \mathrm{P}<0.05 ;{ }^{* *} \mathrm{P}<0.01$. $\mathrm{Bn}$, benign tumor; $\mathrm{Bd}$, borderline tumor; CSTB, cystatin B; GEO, Gene Expression Omnibus; hsa, homo sapiens; Mg, malignant tumor; miR, microRNA.

differed from patients with miR-143-3p information, the expression of CSTB mRNA and miR-143-3p was validated in the same freshly isolated ovarian tissue from the same patient; correlation analysis of these transcripts was performed.

CSTB mRNA expression levels were significantly increased in malignant tumors than in normal ovarian tissue, and benign and borderline tumors $(\mathrm{P}<0.01$; Fig. 1D), which supported the findings of our previous study (3). On the contrary, miR-143-3p was downregulated in malignant tumors compared with normal ovarian tissues and benign tumors $(\mathrm{P}<0.05$; Fig. 1E). Spearman correlation analysis revealed a significant negative correlation between the expression levels of miR-143-3p and CSTB mRNA ( $\mathrm{r}=-0.362, \mathrm{P}<0.05$; Fig. 1F).

miR-143-3p directly binds to the 3'-UTR of CSTB. To determine whether miR-143-3p directly binds to the $3^{\prime}$-UTR of CSTB (Fig. 2A), we cloned two CSTB 3'-UTR plasmids: CSTB-3UTR-wt and CSTB-3UTR-mut. The predicted binding site and mutant site of 3'-UTR (Fig. 2B) were confirmed by sequencing analysis. The luciferase activity in 293T cells was measured after co-transfection of CSTB-3'UTR-containing plasmids with miR-143-3p mimics or miR-NC by a dual-luciferase reporter assay. Transfection of cells with CSTB
3'-UTR-wt and miR-143-3p revealed a significant reduction in the luciferase activity compared with the corresponding control $(\mathrm{P}<0.01)$. However, the cells expressing the mutated CSTB 3'-UTR-mut demonstrated no marked alterations in luciferase activity, suggesting that mutations in the $3^{\prime}$ UTR of CSTB abrogated the binding to the miR-143-3p (Fig. 2C). These findings indicate that miR-143-3p can directly target CSTB.

CSTB is regulated by miR-143-3p in OC cells. To further confirm that CSTB is a target of miR-143-3p, gain-of-function and loss-of-function approaches were applied. The transfection efficiency of miR-143-3p mimics and inhibitors in OVCAR-3 cells was evaluated. A significant increase and decrease in miR-143-3p expression were detected by RT-qPCR aftercells were transfected with miR-143-3p mimics of inhibitors (anti-miR-143) for $12 \mathrm{~h}$, respectively, compared with the corresponding NC group (Fig. S1A and B). The transfection efficiency of mimics was further detected by fluorescence microscopy at $24 \mathrm{~h}$ post-transfection and $\sim 90 \%$ of cells were Cy3-mimic positive (Fig. S1C). Additionally, the expression of CSTB mRNA and protein were examined by RT-qPCR and western blot analyses of OVCAR-3 cells transfected with miR-143-3p mimics 
A

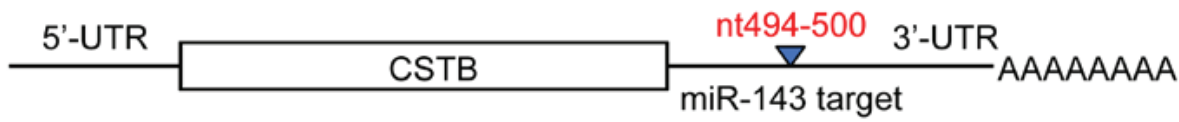

B

CSTB-3UTR-wt: 5' . . UGCACUUGUGAUCCUAAAAUAAGCUUCAUCUCC. . . 3'

hsa-miR-143-3p: $3^{\prime}$

$\| 11111$

CUCGAUGUCACGAAGUAGAGU $2^{\prime}$

CSTB-3UTR-mut: 5 ' . . UGCACUUGUGAUCCUAAAAUAAgCUUGUAgGUC . . . 3'

C

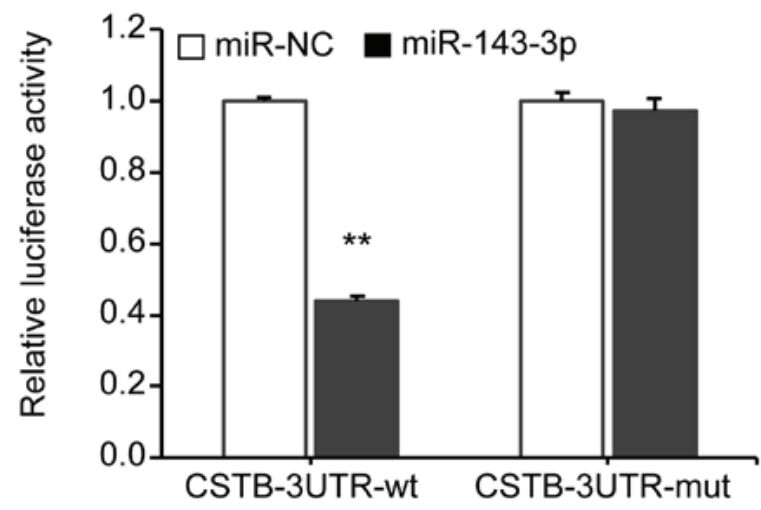

Figure 2. Interaction between CSTB and miR-143-3p transcripts. (A) Illustration of the structure of CSTB with the predicted binding site of miR-143-3p to the 3'-UTR at the position of nucleotide (nt) 494-500 as indicated by a reverse triangle. (B) The partial sequence of CSTB 3'-UTR-wt and the sequence of hsa-miR-143-3p. Nucleotides in red in CSTB-3UTR-wt indicated complimentarity to miR-143-3p, and was mutated in CSTB-3'-UTR-mut plasmid. (C) Dual-luciferase reporter assay. 293T cells were cultured in 24-well plates and cotransfected with CSTB-3UTR-wt or CSTB-3UTR-mut plasmid, and miR-143-3p mimics or miR-NC. Experiments were repeated three times. Data are presented as the mean \pm standard error of the mean. ${ }^{* *} \mathrm{P}<0.01 \mathrm{vs} . \mathrm{miR}-\mathrm{NC}$. 3'-UTR, 3'-untranslated region; CSTB, cystatin B; hsa, homo sapiens; miR, microRNA; NC, negative control.

A

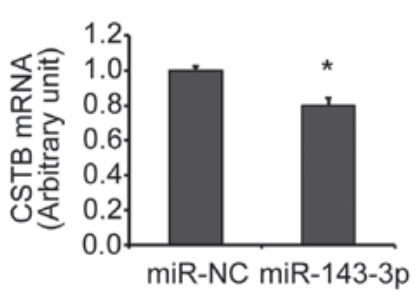

D

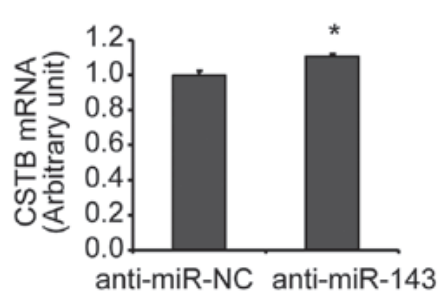

B

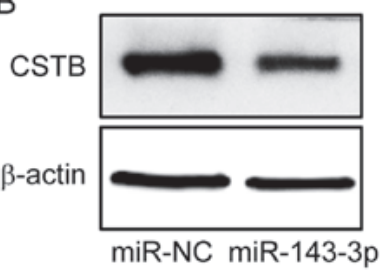

$\mathrm{E}$

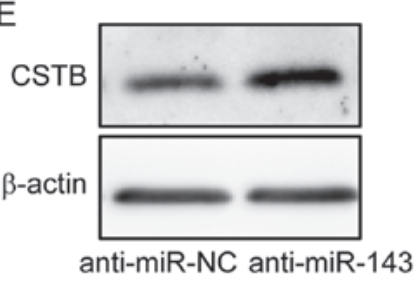

C

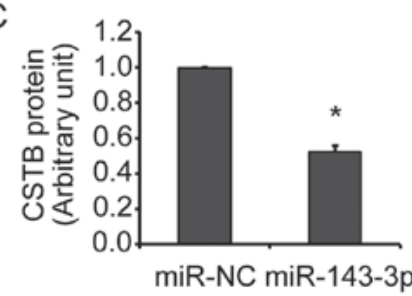

$\mathrm{F}$

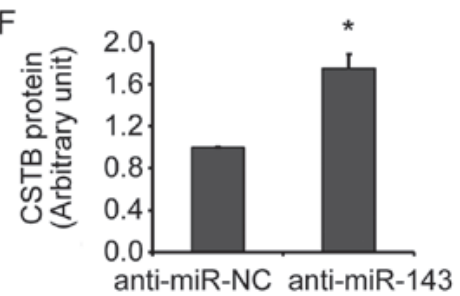

Figure 3. Effects of miR-143-3p on CSTB expression in ovarian cancer cells. OVCAR-3 cells were transfected with miR-143-3p mimics, anti-miR-143 or their corresponding NC. CSTB mRNA was detected by reverse transcription-quantitative PCR at $48 \mathrm{~h}$ post-transfection and protein was detected by western blot at 72 h. (A) CSTB mRNA detection after transfection with miR-143-3p mimics. (B) CSTB protein expression after transfection with miR-143-3p mimics (C) Densitometry analysis of CSTB expression in cells transfected with miR-143-3p mimics compared with miR-NC. (D) CSTB mRNA detection after transfection with anti-miR-143. (E) CSTB protein expression after transfection with anti-miR-143. (F) Densitometry analysis of CSTB expression in cells transfected with anti-miR-143 compared with anti-miR-NC. $n=3$; ${ }^{*} \mathrm{P}<0.05$ vs. miR-NC or anti-miR-NC. CSTB, cystatin B; miR, microRNA; NC, negative control.

or inhibitors. CSTB expression was significantly downregulated at the mRNA (Fig. 3A) and protein (Fig. 3B and C) levels after miR-143-3p mimics transfection. Silencing of miR-143-3p by anti-miR-143 resulted in a significant increase in CSTB expression at the mRNA (Fig. 3D) and protein (Fig. 3C and D) levels in OVCAR-3 cells. These results indicated that miR-143-3p may negatively regulate CSTB expression in OC cells.
miR-143-3p expression is regulated by the TGF- $\beta$ signaling pathway. As our previous findings indicated that CSTB is mediated by the TGF- $\beta$ signaling pathway in epithelial OC cells (3) and the present study revealed that miR-143-3p can negatively regulate CSTB expression, whether the TGF- $\beta$ signaling pathway affects miR-143-3p expression was investigated. RT-qPCR revealed that primary miR-143-3p 
A

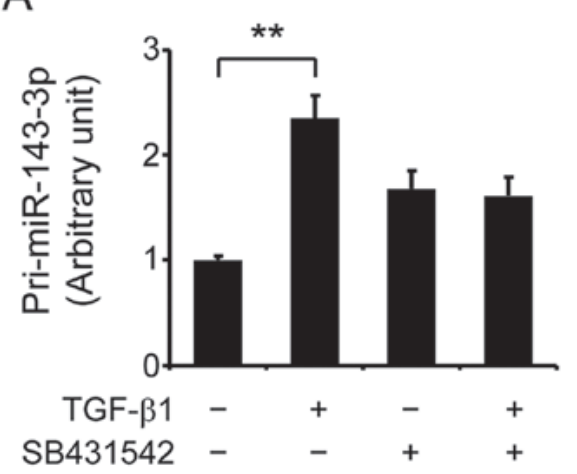

B

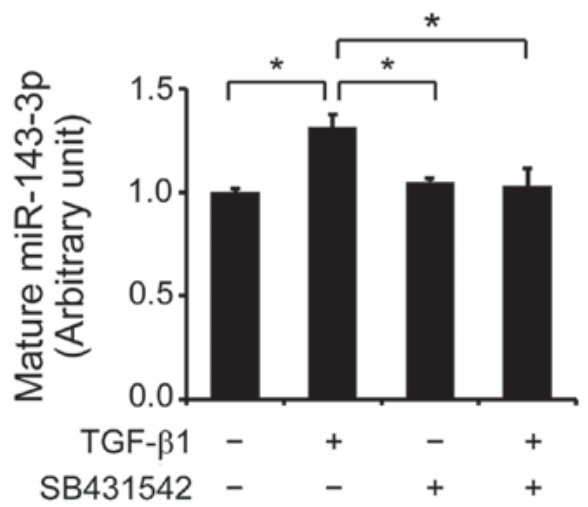

Figure 4. Effects of TGF- $\beta 1$ on pri-miR-143-3p and mature miR-143-3p expression in ovarian cancer cells. OVCAR-3 cells were treated with $10 \mathrm{ng} / \mathrm{ml}$ TGF- $\beta 1$ along for $3 \mathrm{~h}$ or pre-treated with the inhibitor (SB431542) of the TGF- $\beta 1$ type I receptor for $30 \mathrm{~min}$, followed by $10 \mathrm{ng} / \mathrm{ml}$ TGF- $\beta 1$ treatment for 3 h. (A) Detection of pri-miR-143-3p expression by RT-qPCR. (B) Detection of mature miR-143-3p expression by RT-qPCR. $\mathrm{n}=3$; ${ }^{*} \mathrm{P}<0.05$, ${ }^{* * *} \mathrm{P}<0.01$. miR, microRNA; pri, primary; RT-qPCR, reverse transcription-quantitative polymerase chain reaction; TGF- $\beta 1$, transforming growth factor- $\beta 1$.

expression was significantly increased after treatment with $10 \mathrm{ng} / \mathrm{ml}$ TGF- $\beta 1$ in OVCAR-3 cells compared with the control $(\mathrm{P}<0.01$; Fig. $4 \mathrm{~A})$. The effects of TGF- $\beta 1$ were partially abolished by the blocking of the TGF- $\beta$ type I receptor using its inhibitor SB431542. In addition, an increase in the levels of mature miR-143-3p was observed following TGF- $\beta 1$ treatment, which was significantly abolished in the presence of SB431542 ( $\mathrm{P}<0.05$; Fig. 4B).

High levels of CSTB expression are associated with poor OS in patients with ovarian malignant tumors. Overexpression of CSTB in OC was detected in two microarray datasets from the Oncomine database. The median value of CSTB mRNA expression was calculated from two datasets. CSTB mRNA levels were higher in ovarian serous carcinoma than normal ovarian surface epithelium in the Lu dataset (Reporter ID: 35816_at; Fig. 5A) and in the Bonome dataset (Reporter ID: 201201_at; Fig. 5B). Furthermore, OS analysis was performed using the Kaplan-Meier Plotter dataset. Increased CSTB mRNA expression levels were associated with poor OS in patients with OC (Fig. 5C).

Knockdown of CSTB inhibits OC cell proliferation. To determine the functional effects of CSTB on the biological behaviors of OC cells, loss-of-function experiments were applied. A total of three siRNAs specific to human CSTB (CSTB-siRNA) were synthesized; transfection efficiency was determined prior to subsequent experiments. Knockdown of CSTB expression at the mRNA (Fig. S2A and B) and protein (Fig. S2C and D) levels was confirmed by RT-qPCR and western blotting in OVCAR-3 (Fig. S2A and C) and SK-OV-3 (Fig. S2B and D) cells, respectively. Increased transfection efficiency was determined in cells transfected with the second CSTB-siRNA (si-CSTB-2), which was then used for the subsequent experiments and to generate shRNAs. Additionally, a cell viability assay was performed after OVCAR-3 cells were transfected with si-CSTB-2. The results revealed that knockdown of CSTB expression by CSTB-siRNA significantly decreased cell proliferation at 48 and $72 \mathrm{~h}$ compared with the NC group (Fig. S3A). The suppression of CSTB by CSTB-siRNA appeared to have arrested the cell cycle in OVCAR-3 cells at G2/M phase as detected by flow cytometry (Fig. S3B and C).
Similar results were observed in OVCAR-3 cells infected with CSTB-shRNA lentivirus. Knockdown of CSTB protein was confirmed by western blotting (Fig. 6A and B). Cell proliferation was significantly reduced following lentiviral transduction of CSTB-shRNA compared with the NC group at $72 \mathrm{~h}$ (Fig. 6C). In addition, the suppression of CSTB notably increased the number of OVCAR-3 cells at G2/M phase compared with negative controls (Fig. 6D-F).

Knockdown of CSTB induces OC cell apoptosis. Apoptotic cells were detected by flow cytometry after infecting OVCAR-3 cells with CSTB-shRNA or sh-NC via lentivirus for $48 \mathrm{~h}$ (Fig. 7A and B). The cell population in the bottom right of quadrant graphs represented the early apoptotic cells which were significantly increased in the CSTB-shRNA group compared with the NC group (Fig. 7C). Additionally, an increase in the expression of pro-apoptotic protein Bax was observed by western blotting in OVCAR-3 cells infected with CSTB-shRNA lentivirus (Fig. 7D).

\section{Discussion}

CSTB is one of the endogenous inhibitors of lysosomal cysteine proteases, and was reported to be dysregulated in several types of cancer $(3,8-11)$. Our previous study showed that CSTB is overexpressed in human EOC and is mediated by the TGF- $\beta$ signaling pathway (3). To the best of our knowledge, the present study is the first to demonstrate that CSTB could be downregulated by miR-143-3p, whereas miR-143-3p was upregulated by TGF- $\beta 1$. Our findings suggested the existence of the TGF- $\beta / \mathrm{miR}-143-3 \mathrm{p} / \mathrm{CSTB}$ axis in OC cells. Furthermore, CSTB was proposed to function as an oncogene, which affected OC cell proliferation; the expression levels of CSTB were associated with the OS of patients with OC.

Upregulated CSTB expression has also been detected in ovarian clear cell carcinoma (29). Similarly, overexpression of CSTB has been observed in other malignant tumors, such as hepatocellular, bladder and colorectal cancers $(9,30,31)$. CSTB ablation was determined to retard breast tumor growth in a mouse model (32). Our previous study revealed that overexpression of CSTB is associated with the clinicopathological features of EOC (3). In the present study, upregulated CSTB expression 

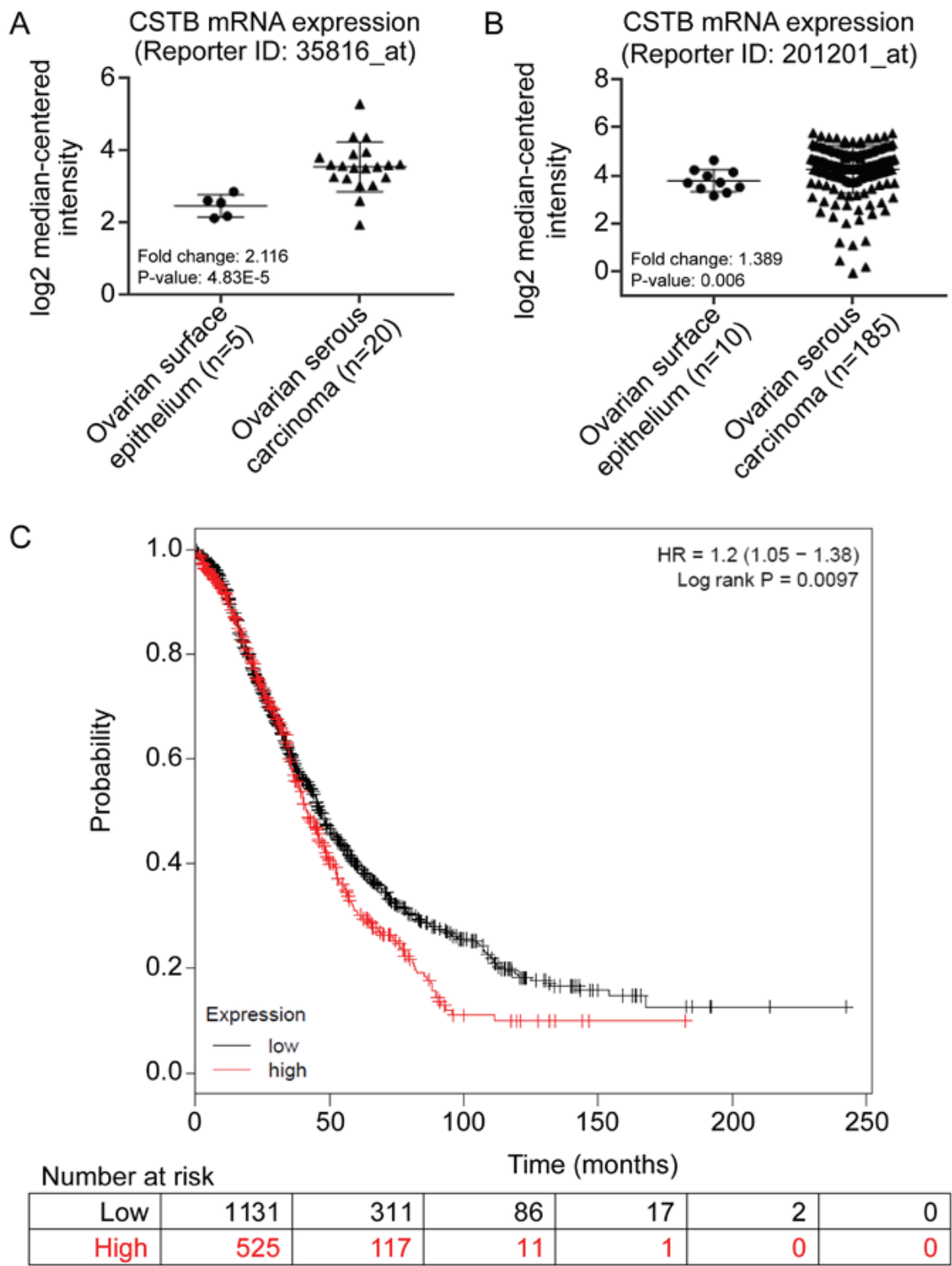

Figure 5. Expression of CSTB mRNA in the ovary and the plot of OS. (A) The data of CSTB mRNA expression in human ovarian tissue were obtained from the microarray dataset (Reporter ID: 35816_at) in the Oncomine database. (B) The data of CSTB mRNA expression in human ovarian tissue were obtained from the microarray dataset (Reporter ID: 201201_at) in the Oncomine database. (C) OS plot. The OS data of patients with ovarian cancer in the low and high CSTB expression groups were obtained from the Kaplan-Meier Plotter datasets. Case number at risk was indicated. CSTB, cystatin B; HR, hazard ratio; OS, overall survival.

was proposed to be associated with the OS of OC patients. It has been shown that CSTB is a prognostic factor, while high serum levels of CSTB were linkted to increased risk of mortality in patients with colorectal cancer (31).

As serous carcinoma, arising from the ovarian surface epithelium and/or fallopian tube epithelium, is the most common type of OC (33), the current study analyzed the expression of CSTB mRNA and miR-143-3p between normal ovary controls and ovarian serous carcinoma from the public GEO database. Our findings were verified in freshly isolated human ovarian serous tumors from our hospital; functional assays using the serous type of human OC cell lines, such as OVCAR-3 and SK-OV-3, were also conducted. Using a loss-of-function approach to knockdown CSTB expression in EOC cells, we found that downregulated CSTB expression significantly inhibited cell proliferation. CSTB-shRNA significantly increased the number of cells in early apoptosis. Elevated pro-apoptotic protein Bax expression was also observed in OVCAR-3 cells after CSTB downregulation. The effects of CSTB downregulation on OC cell proliferation the cell cycle appeared to be more pronounced than the effects of cell apoptosis. These data indicated that CSTB serves a role in the behavior of OC cells and suggest a potential role of CSTB in targeting OC. Furthermore, a regulatory mechanism has been investigated; in the majority of mammalian cells, miRNAs are major regulators of gene expression, and can affect the expression of numerous oncogenes and tumor suppressor genes $(16,34,35)$. Alterations in miRNA expression contributes to a substantial cell re-organization, and thus, is involved in the pathogenesis of many diseases $(13,15)$.

Using the miRWalk database, miR-143-3p was determined to be a candidate by 5 of 10 prediction programs. The present 
A

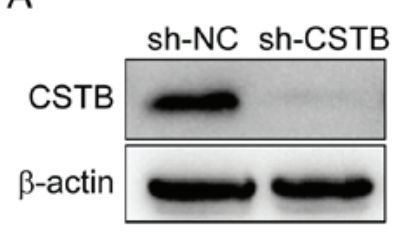

D



$\mathrm{B}$

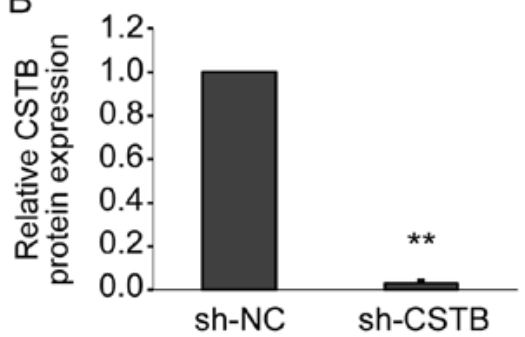

E



C

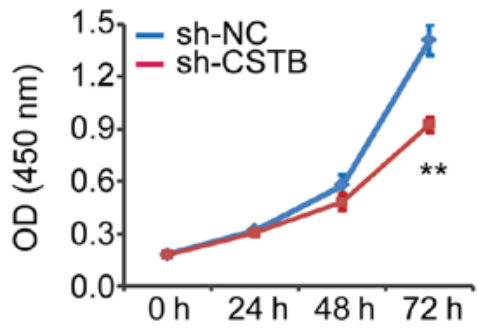

$\mathrm{F}$

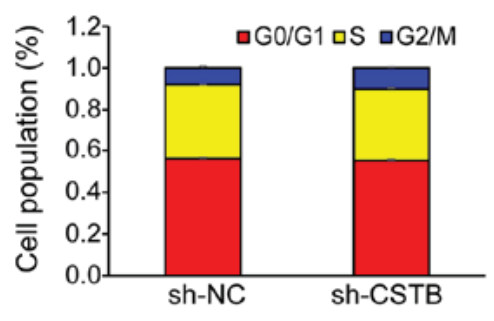

Figure 6. Measurement of OVCAR-3 cell proliferation. (A) Detection of CSTB protein expression by western blotting in OVCAR-3 cells infected with sh-NC or sh-CSTB lentivirus. (B) Densitometry analysis of protein expression ( $\mathrm{n}=3 ;{ }^{* *} \mathrm{P}<0.01$ vs. sh-NC). (C) Determination of cell proliferation in OVCAR-3 cells infected with sh-NC or sh-CSTB by a Cell Counting Kit- 8 assay ( $n=3{ }^{* * *} \mathrm{P}<0.01$ vs. sh-NC). (D) Detection of the sh-NC-infected OVCAR-3 cell cycle by flow cytometry. (E) Detection of the sh-CSTB-infected OVCAR-3 cell cycle by flow cytometry. (F) Cell population in each phase (n=3). CSTB, cystatin B; NC, negative control; sh, short hairpin RNA.

A

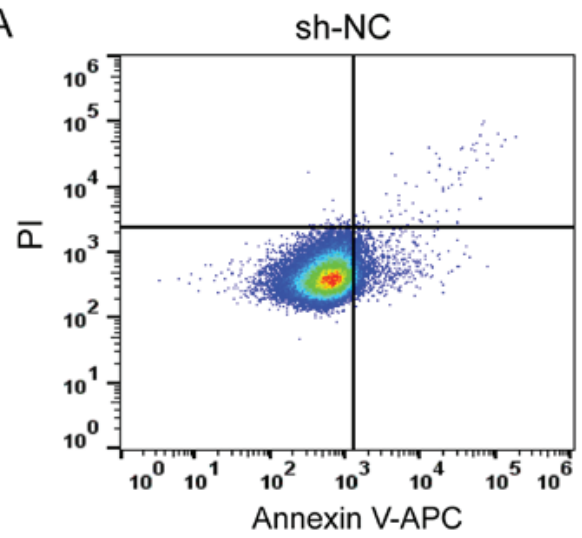

C

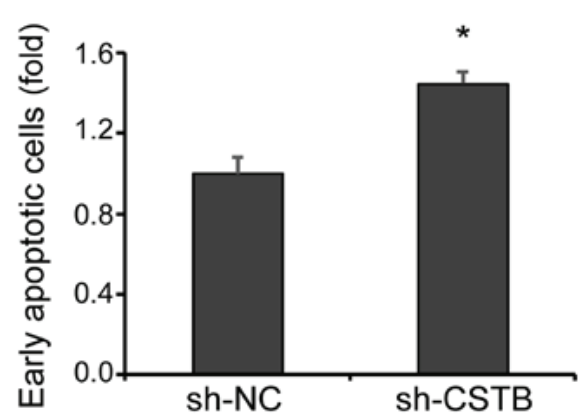

B

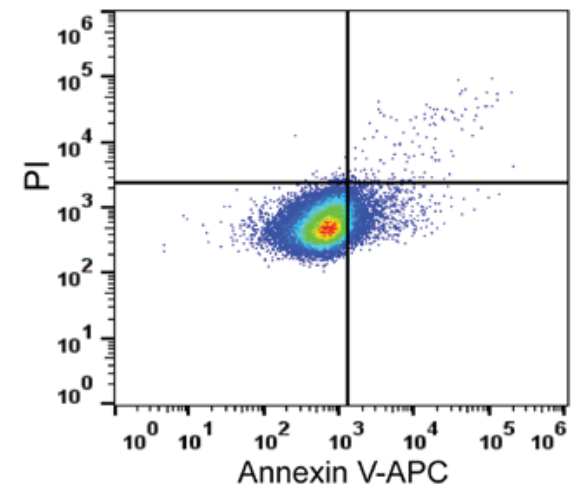

D

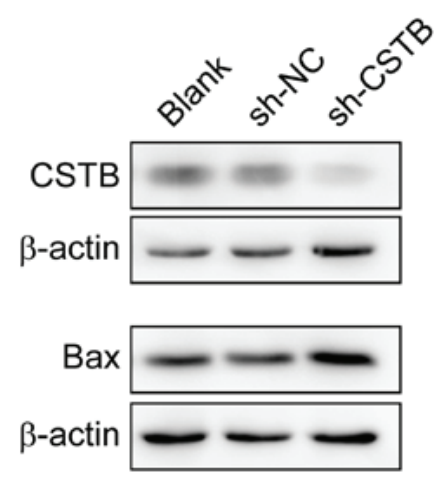

Figure 7. Measurement of OVCAR-3 cell apoptosis. (A) Detection of apoptotic OVCAR-3 cells after sh-NC infection via lentivirus by flow cytometry. (B) Detection of apoptotic OVCAR-3 cells after sh-CSTB via lentivirus by flow cytometry. (C) Fold change of early apoptotic cells between the sh-NC and sh-CSTB groups $\left(\mathrm{n}=3\right.$; $\left.{ }^{*} \mathrm{P}<0.05\right)$. (D) Detection of the expression of CSTB and Bax proteins by western blotting in OVCAR-3 cells infected with sh-NC or sh-CSTB. APC, allophycocyanin; Bax, Bcl-2-associated X protein; CSTB, cystatin B; NC, negative control; sh, short hairpin RNA.

study reported a low level of miR-143-3p expression in OC patients; miR-143-3p negatively correlated with CSTB expression. Treating OC cells with miR-143-3p mimics or inhibitors resulted in a respective decrease and increase in CSTB expression within OC cells. Our findings suggest that CSTB is a target of miR-143-3p. miRNAs can function as suppressors of target mRNA expression by the binding base pairing with a complementary sequence, leading to the degradation of target 
mRNA or the suppression of mRNA translation, resulting in reduced protein expression. An inhibitor of miRNA such as anti-miR-143-3p has the opposite function of miR-143-3p mimics. As it is an antisense RNA and complementary to sense miRNA, the anti-miRNA can inhibit the endogenous miRNA, leading to reduced inhibition of mRNAs mediated by miRs. Upregulation of CSTB protein was detected after anti-miR143-3p treatment, indicating that the suppression of miRNAs can lead to the overexpression of their target transcripts.

Several studies have suggested that miR-143-3p functions as a tumor suppressor. For instance, miR-143-3p inhibits cell proliferation and induces cell apoptosis in esophageal squamous cell carcinoma (36), and suppresses EMT in breast cancer (37). In prostate and bladder cancers, miR-143-3p inhibits cell proliferation and migration $(38,39)$. High-throughput miRNA profiling demonstrated that miR-143-3p is significantly downregulated in OC tissues compared with normal ovarian tissues, while elevated miR-143-3p expression in the OC cell lines OVCAR-3, SK-OV-3 and ES2 significantly reduced their proliferation, migration and invasion (40). Consistently with these studies, our findings indicate that miR-143-3p is dysregulated in OC. Low levels of miR-143-3p may lead to an increase in the expression of CSTB. The present study provides notable evidence that CSTB could be regulated by miR143-3p as suggested by gain-of-function and loss-of-function approaches, indicating the association between miR-143-3p and CSTB in OC.

Our previous study has shown that CSTB expression is mediated by the TGF- $\beta 1$ signaling pathway in OC cells (3); however, the molecular mechanism underlying TGF- $\beta$-mediated CSTB expression remains unknown. The current study demonstrated that TGF- $\beta 1$ increased the expression of primary and mature miR-143-3p. SB431542 (an inhibitor of TGF- $\beta$ type I receptor) markedly increased pri-miR-143-3p expression and partially abolished the effects of TGF- $\beta$, whereas the TGF- $\beta 1$-induced upregulation of mature miR-143-3p was significantly abolished following SB431542 exposure. A single primary miRNA may contain one to six miRNA hairpin loop structures comprising 70 nucleotides each, which can be cleaved by Drosha (ribonuclease III enzyme) together with DiGeorge Syndrome Critical Region 8 to form precursor miRNAs in the nucleus. Subsequently, precursor miRNAs are transported from the nucleus into the cytoplasm by Exportin- 5 and cleaved by Dicer to generate double-stranded mature miRNA $(12,14,16)$; TGF- $\beta$ may influence the processing of miRNAs (19). There is a CArG box and a Smad binding element located in the 580-bp enhancer region of miR-143-3p, which fully respond to TGF- $\beta 1$ stimulation upon the binding of the Smad3/Smad4 to the enhancer region (41). Similarly, in vascular smooth muscle cells, TGF- $\beta$ binds to miR-143 via the CArG box (42). These findings suggest the existence of a TGF- $\beta /$ miR-143-3p/CSTB axis in which the levels of miR-143-3p are upregulated after TGF- $\beta 1$ stimulation, and interacts with the 3'-UTR of CSTB mRNA, leading to the downregulation of CSTB expression.

In conclusion, CSTB is overexpressed and negatively correlated with miR-143-3p expression in human EOC. High levels of CSTB expression were associated with the poor OS of patients with OC. Suppression of CSTB was demonstrated to inhibit OC cell proliferation and induce apoptosis. Mature miR-143-3p directly bound the 3'UTR of CSTB, leading to a decrease in CSTB expression in OC cells, which is regulated by TGF- $\beta 1$. Our findings suggested the therapeutic potential of targeting the TGF- $\beta / \mathrm{miR}-143-3 \mathrm{p} / \mathrm{CSTB}$ axis for treating patients with $\mathrm{OC}$.

\section{Acknowledgements}

Not applicable.

\section{Funding}

The present study was supported by grants from the National Natural Science Foundation of China (grant no. 81872121), the Natural Science Foundation of Shanghai (grant no. 17ZR1404100), the Shanghai Municipal Commission of Health and Family Planning (grant no. 201640287 to G.X.), and a grant from Jinshan District Commission of Health and Family Planning (grant no. JSKJ-KTMS-2016-01 to W.G.).

\section{Availability of data and materials}

All data generated or analyzed during this study are included in this published article.

\section{Authors' contributions}

WG performed the majority of experiments, analyzed the data, generated figures and wrote the manuscript. XW conducted the siRNA experiments. QL, JZ, WR were involved in the experiments and performed bioinformatics analysis. GX made substantial contributions to the design of the study, conducted data analysis and figure generation, and wrote the manuscript. All authors read and approved the final manuscript.

\section{Ethics approval and consent to participate}

The present study was approved by the Ethics Committee of Jinshan Hospital (approval no. E-2013-018-01; Shanghai, China).

\section{Patient consent for publication}

Not applicable.

\section{Competing interests}

The authors declare that they have no competing interests.

\section{References}

1. Turk V, Stoka V and Turk D: Cystatins: Biochemical and structural properties, and medical relevance. Front Biosci 13: 5406-5420, 2008.

2. Žerovnik E: Putative alternative functions of human stefin B (cystatin B): Binding to amyloid-beta, membranes, and copper. J Mol Recognit 30: 30, 2017.

3. Wang X, Gui L, Zhang Y, Zhang J, Shi J and Xu G: Cystatin B is a progression marker of human epithelial ovarian tumors mediated by the TGF- $\beta$ signaling pathway. Int J Oncol 44: 1099-1106, 2014.

4. Bray F, Ferlay J, Soerjomataram I, Siegel RL, Torre LA and Jemal A: Global cancer statistics 2018: GLOBOCAN estimates of incidence and mortality worldwide for 36 cancers in 185 countries. CA Cancer J Clin 68: 394-424, 2018. 
5. Siegel RL, Miller KD and Jemal A: Cancer statistics, 2019. CA Cancer J Clin 69: 7-34, 2019.

6. Maher K, Jerič Kokelj B, Butinar M, Mikhaylov G, Manček-Keber M, Stoka V, Vasiljeva O, Turk B, Grigoryev SA and Kopitar-Jerala N: A role for stefin B (cystatin B) in inflammation and endotoxemia. J Biol Chem 289: 31736-31750, 2014.

7. Lalioti MD, Scott HS, Buresi C, Rossier C, Bottani A, Morris MA, Malafosse A and Antonarakis SE: Dodecamer repeat expansion in cystatin B gene in progressive myoclonus epilepsy. Nature 386: 847-851, 1997

8. Gashenko EA, Lebedeva VA, Brak IV, Tsykalenko EA, Vinokurova GV and Korolenko TA: Evaluation of serum procathepsin B, cystatin B and cystatin C as possible biomarkers of ovarian cancer. Int J Circumpolar Health 72: 21215, 2013.

9. Feldman AS, Banyard J, Wu CL, McDougal WS and Zetter BR: Cystatin B as a tissue and urinary biomarker of bladder cancer recurrence and disease progression. Clin Cancer Res 15: 1024-1031, 2009.

10. Lee MJ, Yu GR, Park SH, Cho BH, Ahn JS, Park HJ, Song EY and Kim DG: Identification of cystatin B as a potential serum marke in hepatocellular carcinoma. Clin Cancer Res 14: 1080-1089, 2008.

11. Shiraishi T, Mori M, Tanaka S, Sugimachi K and Akiyoshi T: Identification of cystatin B in human esophageal carcinoma using differential displays in which the gene expression is related to lymph-node metastasis. Int J Cancer 79: 175-178, 1998.

12. Treiber T, Treiber $\mathrm{N}$ and Meister G: Regulation of microRNA biogenesis and its crosstalk with other cellular pathways. Nat Rev Mol Cell Biol 20: 5-20, 2019

13. Bartel DP: MicroRNAs: Target recognition and regulatory functions. Cell 136: 215-233, 2009.

14. Ha M and Kim VN: Regulation of microRNA biogenesis. Nat Rev Mol Cell Biol 15: 509-524, 2014.

15. van Kouwenhove M, Kedde M and Agami R: MicroRNA regulation by RNA-binding proteins and its implications for cancer Nat Rev Cancer 11: 644-656, 2011.

16. Zhang L, Nadeem L, Connor K and Xu G: Mechanisms and therapeutic targets of microRNA-associated chemoresistance in epithelial ovarian cancer. Curr Cancer Drug Targets 16: 429-441, 2016.

17. Behbahani GD, Ghahhari NM, Javidi MA, Molan AF, Feizi N and Babashah S: MicroRNA-mediated post-transcriptional regulation of epithelial to mesenchymal transition in cancer. Patho Oncol Res 23: 1-12, 2017.

18. Schetter AJ, Heegaard $\mathrm{NH}$ and Harris CC: Inflammation and cancer: Interweaving microRNA, free radical, cytokine and p53 pathways. Carcinogenesis 31: 37-49, 2010.

19. Ottley E and Gold E: microRNA and non-canonical TGF- $\beta$ signalling: Implications for prostate cancer therapy. Crit Rev Oncol Hematol 92: 49-60, 2014.

20. Shi Y and Massagué J: Mechanisms of TGF-beta signaling from cell membrane to the nucleus. Cell 113: 685-700, 2003.

21. Attisano L and Wrana JL: Signal transduction by the TGF-beta superfamily. Science 296: 1646-1647, 2002.

22. Schmierer B and Hill CS: TGFbeta-SMAD signal transduction: Molecular specificity and functional flexibility. Nat Rev Mol Cell Biol 8: 970-982, 2007.

23. Derynck R and Zhang YE: Smad-dependent and Smadindependent pathways in TGF-beta family signalling. Nature 425 : $577-584,2003$

24. Sun W, Gui L, Zuo X, Zhang L, Zhou D, Duan X, Ren W and Xu G: Human epithelial-type ovarian tumour marker beta-2-microglobulin is regulated by the TGF- $\beta$ signaling pathway. $\mathrm{J}$ Transl Med 14: 75, 2016.

25. Matsumoto T, Yokoi A, Hashimura M, Oguri Y, Akiya M and Saegusa M: TGF- $\beta$-mediated LEFTY/Akt/GSK-3 $\beta /$ Snail axis modulates epithelial-mesenchymal transition and cancer stem cell properties in ovarian clear cell carcinomas. Mol Carcinog 57: 957-967, 2018
26. Lu KH, Patterson AP, Wang L, Marquez RT, Atkinson EN, Baggerly KA, Ramoth LR, Rosen DG, Liu J, Hellstrom I, et al: Selection of potential markers for epithelial ovarian cancer with gene expression arrays and recursive descent partition analysis. Clin Cancer Res 10: 3291-3300, 2004.

27. Bonome T, Levine DA, Shih J, Randonovich M,Pise-Masison CA, Bogomolniy F, Ozbun L, Brady J, Barrett JC, Boyd J, et al: A gene signature predicting for survival in suboptimally debulked patients with ovarian cancer. Cancer Res 68: 5478-5486, 2008.

28. Livak KJ and Schmittgen TD: Analysis of relative gene expression data using real-time quantitative PCR and the 2(-Delta Delta C(T)) $\mu$ ethod. Methods 25: 402-408, 2001.

29. Takaya A, Peng WX, Ishino K, Kudo M, Yamamoto T, Wada R, Takeshita $\mathrm{T}$ and Naito Z: Cystatin B as a potential diagnostic biomarker in ovarian clear cell carcinoma. Int J Oncol 46: 1573-1581, 2015.

30. Lin YY, Chen ZW, Lin ZP, Lin LB, Yang XM, Xu LY and Xie Q: Tissue Levels of Stefin A and Stefin B in Hepatocellular Carcinoma. Anat Rec (Hoboken) 299: 428-438, 2016.

31. Kos J, Krasovec M, Cimerman N, Nielsen HJ, Christensen IJ and Brünner N: Cysteine proteinase inhibitors stefin A, stefin B, and cystatin $\mathrm{C}$ in sera from patients with colorectal cancer: Relation to prognosis. Clin Cancer Res 6: 505-511, 2000.

32. Butinar M, Prebanda MT, Rajkovic J, Jerič B, Stoka V, Peters C, Reinheckel T, Krüger A, Turk V, Turk B, et al: Stefin B deficiency reduces tumor growth via sensitization of tumor cells to oxidative stress in a breast cancer model. Oncogene 33: 3392-3400, 2014.

33. Jelovac D and Armstrong DK: Recent progress in the diagnosis and treatment of ovarian cancer. CA Cancer J Clin 61: 183-203, 2011.

34. Cheng CJ, Bahal R, Babar IA, Pincus Z, Barrera F, Liu C, Svoronos A, Braddock DT, Glazer PM, Engelman DM, et al: MicroRNA silencing for cancer therapy targeted to the tumour microenvironment. Nature 518: 107-110, 2015.

35. Guo H, Ingolia NT, Weissman JS and Bartel DP: Mammalian microRNAs predominantly act to decrease target mRNA levels. Nature 466: 835-840, 2010.

36. He Z, Yi J, Liu X, Chen J, Han S, Jin L, Chen L and Song H: MiR-143-3p functions as a tumor suppressor by regulating cell proliferation, invasion and epithelial-mesenchymal transition by targeting QKI-5 in esophageal squamous cell carcinoma. Mol Cancer 15: 51, 2016.

37. Zhai L, Ma C, Li W, Yang S and Liu Z: miR-143 suppresses epithelial-mesenchymal transition and inhibits tumor growth of breast cancer through down-regulation of ERK5. Mol Carcinog 55: 1990-2000, 2016.

38. Xu B, Niu X, Zhang X, Tao J, Wu D, Wang Z, Li P, Zhang W, Wu H, Feng N, et al: miR-143 decreases prostate cancer cells proliferation and migration and enhances their sensitivity to docetaxel through suppression of KRAS. Mol Cell Biochem 350: 207-213, 2011

39. Song T, Zhang X, Wang C, Wu Y, Dong J, Gao J, Cai W and Hong B: Expression of miR-143 reduces growth and migration of human bladder carcinoma cells by targeting cyclooxygenase- 2 . Asian Pac J Cancer Prev 12: 929-933, 2011.

40. Shi H, Shen H, Xu J, Zhao S, Yao S and Jiang N: MiR-143-3p suppresses the progression of ovarian cancer. Am J Trans1 Res 10: 866-874, 2018.

41. Long X and Miano JM: Transforming growth factor-beta1 (TGF-beta1) utilizes distinct pathways for the transcriptional activation of microRNA $143 / 145$ in human coronary artery smooth muscle cells. J Biol Chem 286: 30119-30129, 2011.

42. Davis-Dusenbery BN, Chan MC, Reno KE, Weisman AS, Layne MD, Lagna G and Hata A: down-regulation of Kruppel-like factor-4 (KLF4) by microRNA-143/145 is critical for modulation of vascular smooth muscle cell phenotype by transforming growth factor-beta and bone morphogenetic protein 4. J Biol Chem 286: 28097-28110, 2011. 